\title{
Taponamiento uterino artesanal con condón e innovador sistema de drenaje en el manejo de hemorragia posparto: a propósito de un caso
}

\author{
Drs. Aida Lara, ${ }^{1}$ Hecna Carrillo, ${ }^{1}$ Mayrin Martinez, ${ }^{1}$ Jesús Veroes. ${ }^{2}$
}

\section{RESUMEN}

La hemorragia posparto se encuentra dentro de las complicaciones obstétricas más frecuentes y con mayor tasa de mortalidad. La atonía uterina es una de sus principales causas. Ante el fracaso del manejo médico, es de importancia desarrollar opciones terapéuticas no invasivas y menos mórbidas como el taponamiento uterino. En este reporte, se presenta un caso de atonía uterina manejada con taponamiento uterino artesanal con condón, al cual se le incorpora un sistema de drenaje para cuantificar pérdidas hemáticas con el balón in situ, con control satisfactorio de la hemorragia.

Palabras clave: Taponamiento uterino, Hemorragia posparto, Atonía uterina, Taponamiento artesanal, Taponamiento con drenaje.

\section{SUMMARY}

Postpartum hemorrhage is among the most frequent obstetric complications and with the highest mortality rate. Uterine atony is one of its main causes. Given the failure of medical management, non-invasive and less morbid therapeutic options such as uterine tamponade are of importance. In this report, we present a case of uterine atony managed with artisanal uterine tamponade with a condom, to which a drainage system is incorporated to quantify blood loss with the balloon in situ with satisfactory bleeding control.

Keywords: Uterine tamponade, Postpartum hemorrhage, Uterine atony, Artisanal tamponade, Drainage tamponade.

\section{INTRODUCCIÓN}

La hemorragia posparto (HPP) es una de las principales causas de mortalidad materna en países de bajos ingresos. Anualmente, más de 1,5 millones de mujeres tienen complicaciones relacionadas con la hemorragia durante el embarazo y el posparto (1). Existen muchas causas de HPP, siendo la atonía uterina la más común (2).

\footnotetext{
${ }^{1}$ Ginecobstetra. Maternidad "Concepción Palacios". Caracas, Universidad Central de Venezuela. ${ }^{2}$ Ginecobstetra, Medicina Materno-Fetal. Maternidad "Concepción Palacios". Caracas, Universidad Central de Venezuela.
}

La atonía uterina se maneja inicialmente con terapia médica con una variedad de agentes uterotónicos. De acuerdo con el Colegio Americano de Obstetras y Ginecólogos (3), en su boletín de práctica de HPP, publicado en 2006, cuando los uterotónicos no pueden causar contracción uterina sostenida y control satisfactorio de la hemorragia después del parto vaginal, el taponamiento del útero puede ser eficaz en la disminución de la hemorragia secundaria a atonía uterina.

El taponamiento con balón intrauterino se recomienda como una intervención de segunda línea en el manejo de la HPP con tasas de eficacia reportadas de $80 \%-$ $100 \%$. Puede ser utilizado como una medida temporal, 


\section{TAPONAMIENTO UTERINO ARTESANAL CON CONDÓN E INNOVADOR SISTEMA DE DRENAJE EN EL MANEJO DE HEMORRAGIA POSPARTO: A PROPÓSITO DE UN CASO}

mientras los pacientes esperan ser transferidos a instalaciones de nivel superior, pero también puede evitar la necesidad de cirugía por completo, como histerectomía, entre otras intervenciones (4).

Algunos de los primeros sistemas de taponamiento reportados fueron con el globo Foley, seguido por el globo catéter hidrostático Rusch, que en dos estudios (el más grande con 22 pacientes) mostraron casi un $60 \%$ de cese de sangrado. Posteriormente, el catéter esofágico Sengstaken - Blakemore se utilizó con un $81 \%$ de éxito. El balón SOS Bakri fue creado con la intención de proporcionar mejores resultados; fue aprobado por la U.S. Food and Drug Administration (FDA) y ha sido evaluado con estudios que incluyen de 2 a 16 pacientes, con efectividad estimada de hasta $80 \%-90 \%$. Más recientemente se coloca en el mercado, el globo "ebb" o "globo reflujo", con un sistema de taponamiento de doble globo, y el globo "BT-Cath" que ofrece un globo con un contorno con forma de útero, para prevenir la perforación (2).

Estos modernos y específicos sistemas de taponamiento uterino comercialmente disponibles, con canal de drenaje de sangrado intrauterino, son costosos y, en consecuencia, resultan inaccesibles en entornos de bajos recursos, donde a menudo es necesario el uso de dispositivos artesanales realizados con sondas de Foley y condones, que son de muy bajo costo (4).

Rathore y cols. (5) realizaron un estudio prospectivo en el hospital de Nueva Delhi, en la India, que incluyó 18 pacientes con HPP no traumática, sin respuesta a tratamiento médico, demostraron que el taponamiento con catéter de Foley y condón es efectivo para controlar la HPP no traumática en el 94,4 \% de los casos.

Sin embargo, estos dispositivos artesanales tienen inherentes desventajas. Al no poseer una vía de drenaje, resulta imposible evaluar las pérdidas reales de sangre uterina una vez realizado el taponamiento.
Además se necesita un hilo estéril para atar el condón al catéter; esto pudiera invertir mayor cantidad de tiempo y admitir riesgo de fuga y desinflado del balón (4).

Mishra y cols. (4), en 2016, publicaron un diseño innovador para superar estas desventajas, sin dejar de ser económico, al cual denominan "globo CG" (CG indica el estado de Chhattisgarh en India, donde fue confeccionado). Requiere un catéter de Foley tamaño 20-22, un condón, el uso de tijeras, dos jeringas de $20 \mathrm{ml}$ y solución salina. Se prepara manualmente, cortando la punta del catéter de Foley y el condón de forma conjunta, creando un orificio de drenaje por encima de un anillo colocado en el extremo distal del dispositivo.

Se presenta a continuación un reporte de caso con propuesta de taponamiento uterino con condón y dispositivo de drenaje, manteniendo las características de una modalidad efectiva, fácil de usar, disponible y económica, realizado en la Maternidad Concepción Palacios, Caracas, Venezuela.

\section{HISTORIA CLÍNICA}

Paciente de 24 años de edad, II Gestas, I Para, sin antecedentes patológicos, quien fue referida a la Maternidad Concepción Palacios en vista de hallazgo ecográfico de ausencia de actividad cardíaca fetal.

Al examen físico presentaba: presión arterial (PA): 110/62 mm Hg, frecuencia cardiaca (FC): $70 \mathrm{lpm}$, frecuencia respiratoria (FR): $19 \mathrm{rpm}$. Cardiopulmonar normal. Abdomen: útero grávido, feto único, cefálico, frecuencia cardiaca fetal (FCF) ausente, movimientos fetales ausentes, dinámica uterina positiva, altura uterina: $32 \mathrm{~cm}$. Ginecológico: genitales externos de aspecto y configuración normal, cuello borrado $70 \%, 6 \mathrm{~cm}$ de dilatación, membranas ovulares íntegras, presentación cefálica, insinuada; pelvis clínicamente suficiente. 
Se plantearon los siguientes diagnósticos de ingreso: 1. Óbito fetal ( 35 semanas +3 días por biometría fetal). 2. Anemia leve $(10,5 \mathrm{~g} / \mathrm{dL})$. Se realizó conducción del trabajo de de parto y amniorrexis oportuna, obteniéndose líquido meconial espeso.

Exámenes de laboratorio de ingreso: hemoglobina (Hb): 10,5 g/dL; hematocrito (Hto): 30,2 \%; leucocitos: $9500 \mathrm{cel} / \mathrm{mm}^{3}$; neutrófilos: $69,4 \%$; linfocitos: $23 \%$; plaquetas: $267000 \mathrm{cel} / \mathrm{mm}^{3}$.

Se asistió parto obteniendo recién nacido masculino, peso al nacer: 2900 gramos, sin signos vitales ni signos de maceración, el alumbramiento fue espontáneo a los 5 minutos, la placenta impresionó completa. Se evidenció sangrado genital abundante, tono uterino ausente, sin evidencia de desgarros ni presencia de restos placentarios en canal de parto. Signos vitales PA: 99/60 mm Hg, FC: 110 lpm, FR: 20 rpm, índice de choque: 1,1 .

Se administró oxigenoterapia, masaje uterino bimanual, 1000 cc de solución 0,9\%, 20 UI de oxitocina vía endovenosa (VEV), misoprostol $800 \mathrm{mcg}$ vía oral, ácido tranexámico $1 \mathrm{~g}$ VEV (metilergonovina no disponible); sin cese del sangrado. Se decidió taponamiento uterino con balón artesanal con drenaje conectado a bolsa recolectora, con un volumen de 1lenado de $500 \mathrm{cc}$ de solución $0,9 \%$. Se evidenció cese del sangrado a los 5 minutos de la inserción. Se colocó compresa en canal vaginal para evitar salida del balón.

La paciente permaneció en observación con vigilancia estricta de signos vitales, altura uterina y gasto a través del drenaje. La altura uterina postaponamiento fue de $24 \mathrm{~cm} ; 50 \mathrm{cc}$ de gasto hemático promedio por hora las primeras 4 horas y luego se hizo imperceptible. Recibió 1 unidad de concentrado globular. El control de laboratorio postransfusional reportó: $\mathrm{Hb}: 9,7 \%$; Hto: $31 \%$; leucocitos: $18000 \mathrm{cel} / \mathrm{mm}^{3}$; neutrófilos: $89 \%$; linfocitos: $10 \%$; plaquetas $496000 \mathrm{cel} / \mathrm{mm}^{3}$.
Veinticuatro horas después del taponamiento, se observó estabilidad clínica y hemodinámica, altura uterina: $21 \mathrm{~cm}$, sin gasto hemático adicional a las 22 horas de la colocación del taponamiento artesanal. Se realizó protocolo de retiro de forma progresiva, extrayendo $100 \mathrm{cc} /$ hora con vigilancia de los signos vitales, sin evidenciar sangrado activo a través de genitales, ni signos de descompensación hemodinámica. La paciente egresó a las 72 horas en buenas condiciones generales.

Para la confección y aplicación del dispositivo de taponamiento uterino artesanal con drenaje se utilizó el siguiente material: equipo de revisión uterina compuesto por una valva vaginal y dos pinzas de Föerster, 1 condón o preservativo, una sonda de Foley $\mathrm{N}^{\mathrm{o}} 12$ y una sonda de Nelaton $\mathrm{N}^{\mathrm{o}} 16$ (ambas de látex), sutura de seda 1, un macrogotero, solución fisiológica $0,9 \%$, bisturí o tijera, campos estériles y bolsa recolectora (Figura 1).

Se procedió a abrir el estuche del condón, sacándolo del mismo sin desplegarlo. Se colocó en este caso una sonda de Foley, se inflo el balón de llenado (Figura

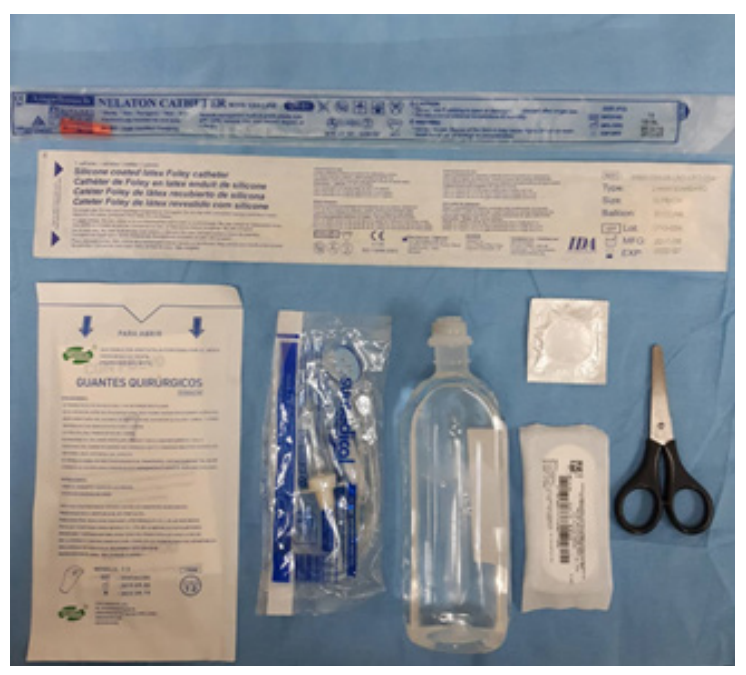

Figura 1. Materiales para la confección del balón artesanal con condón y sistema drenaje. 

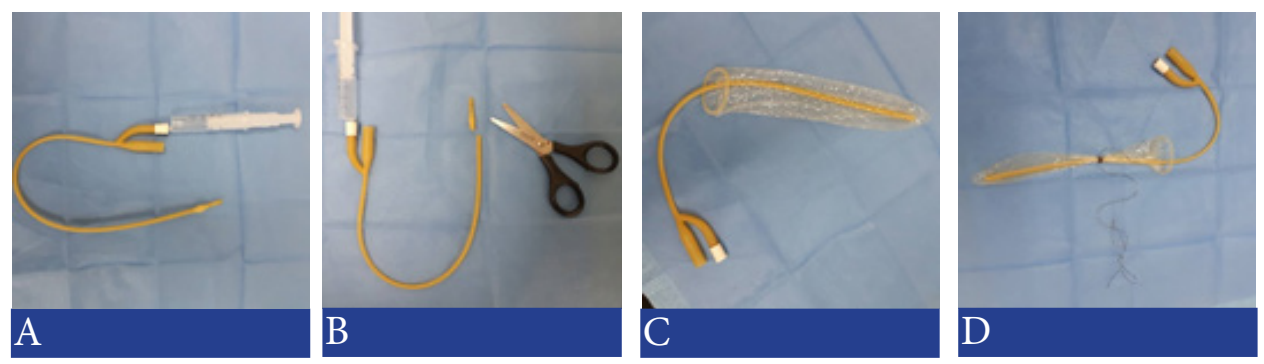

Figura 2. Colocación de condón a la sonda de Foley. A: Sonda de Foley conectada a una jeringa. B: Corte del extremo distal eliminando el balón de llenado. C: Condón desplegado a través del cuerpo de la sonda. D: Nudo continuo a $10 \mathrm{~cm}$ de la punta de la sonda con sutura de seda.

2-A), para luego proceder a cortar el extremo distal, eliminándolo (Figura 2-B). La punta se puso en contacto con el final del reservorio del condón, desplegándolo a través del cuerpo de la sonda (Figura 2-C). Se procedió a medir $10 \mathrm{~cm}$ desde la punta hacia el extremo proximal, realizando un nudo continuo alrededor de la misma con sutura de seda, aplicando vueltas y tensión lo suficientemente fuerte para evitar la salida del líquido y lo suficientemente suave para no obturar la luz del catéter y permitir el llenado (Figura 2-D). Posteriormente, se anexó como futuro sistema de drenaje, una sonda de Nelaton, que se fijó con sutura de seda al dispositivo (Figura 3-A), anudándola a nivel del nudo realizado sobre la superficie del condón y dejando libre el orificio distal del segundo catéter. Se conectó un sistema de macrogotero al agujero de llenado de la sonda del dispositivo con condón, unido a una bolsa de 500 cc de solución fisiológica fijando esta conexión con un nudo fuerte para evitar fugas (Figura 3-B). Se aplicó presión positiva sobre la bolsa de solución y a favor de gravedad para llenar el condón con 500 cc (Figura 3-C). Se obliteró la luz de la sonda de Foley, a través de la cual se realizó el llenado y se colocó una bolsa recolectora para drenaje en la sonda de Nelaton, que quedó localizada por fuera del condón.
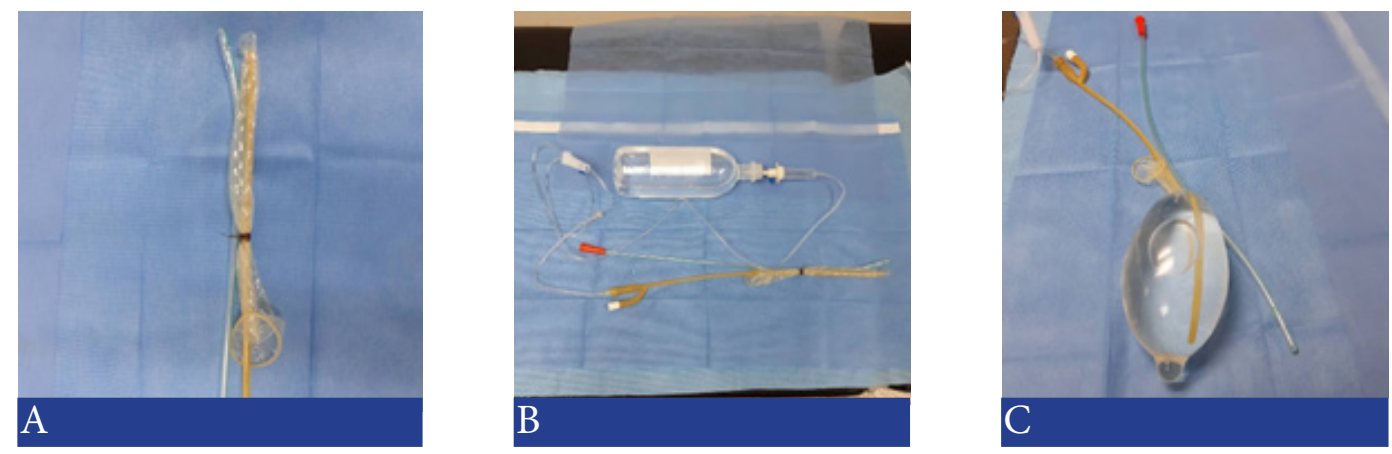

Figura 3. Colocación de sonda de Nelaton y llenado del sistema. A: Sonda de Nelaton unida a la sonda de Foley con condón. B: Frasco de solución de 500 cc, conectada a través de macrogotero a sistema de Foley con condón. C: Sistema de Foley con condón lleno con $500 \mathrm{cc}$ de solución fisiológica. 


\section{DISCUSIÓN}

La atonía uterina se encuentra en las primeras causas de hemorragia posparto; siempre se debe iniciar el manejo estableciendo el grado de choque, estado de conciencia y administrar oxígeno. La primera opción terapéutica consiste en instaurar un manejo activo, con cristaloides o hemoderivados para reemplazar la volemia, masaje uterino bimanual continuo y uterotónicos; de no funcionar estos métodos, se procede a realizar otras técnicas conservadoras como el taponamiento uterino (6 - 9).

La eficacia del taponamiento uterino con condón en el manejo de la HPP no traumática ha sido verificada por Rathore y cols. (5). Las principales medidas de resultado fueron: una tasa de éxito $94,4 \%$ con cantidad media de llenado de $409 \mathrm{ml}$ y un tiempo promedio para controlar el sangrado de $6 \mathrm{~min}$. La duración media del catéter in situ fue de 27,6 horas, resultados comparables a los del caso que se presenta.

Mishra y cols. (4) son pioneros en la publicación de un taponamiento artesanal con sistema de drenaje denominado globo CG, en 2016. Dos años después, ese grupo de trabajo realizó un estudio comparativo entre este dispositivo y el taponamiento convencional con condón; pudieron concluir que ambos son fáciles de usar, factibles y eficaces para control de la HPP, haciendo la observación respecto al dispositivo globo CG, el cual tiene la ventaja de una luz de drenaje central en tiempo real lo que permite la evaluación de la pérdida de sangre $y$, por lo tanto, la corrección y acción temprana respecto al método (10).

En el caso reportado, se presenta un sistema de taponamiento artesanal con un principio similar al propuesto en la confección del globo CG $(4,10)$, pero con una configuración distinta porque el sistema de drenaje se anexa al dispositivo confeccionado con el condón al incorporar un segundo catéter al sistema para obtener el mismo efecto, es decir, drenaje de la pérdida hemática uterina durante la permanencia del balón en el útero. El control de la HPP fue exitoso, permitiendo superar la limitación del taponamiento convencional con condón, por lo que se propone como un método aún más efectivo, que sigue siendo económico, de fácil acceso y aplicación por parte de los proveedores de salud materna, contribuyendo a reducir consecuentemente la morbimortalidad que esta patología produce.

\section{REFERENCIAS}

1. Suarez S, Conde-Agudelo A, Borovac-Pinheiro A, Suarez-Rebling D, Eckardt M, Theron G, et al. Uterine balloon tamponade for the treatment of postpartum hemorrhage: a systematic review and meta-analysis. Am J Obstet Gynecol. 2020; 222(4):293.e1-293.e52. doi: 10.1016/j.ajog.2019.11.1287. Epub 2020 Jan 6. PMID: 31917139.

2. Olsen R, Reisner DP, Benedetti TJ, Dunsmoor$\mathrm{Su}$ RF. Bakri balloon effectiveness for postpartum hemorrhage: a "real world experience". J Matern Fetal Neonatal Med. 2013; 26(17):1720-3. doi: 10.3109/14767058.2013.796354. Epub 2013 May 17. PMID: 23611683.

3. Dildy GA, Belfort MA, Adair CD, Destefano K, Robinson D, Lam G, et al.; ebb Surveillance Study Team. Initial experience with a dual-balloon catheter for the management of postpartum hemorrhage. Am J Obstet Gynecol. 2014; 210(2):136.e1-6. doi: 10.1016/j.ajog.2013.09.015. Epub 2013 Sep 18. PMID: 24055586.

4. Mishra N, Shrivastava C, Agrawal S, Gulabani K. The CG balloon is an innovative condom balloon tamponade for the management of postpartum hemorrhage in low-resource settings. Int J Gynaecol Obstet. 2016; 133(3):377-8. doi: 10.1016/j.ijgo.2015.10.014. Epub 2016 Feb 1. PMID: 26944256.

5. Rathore AM, Gupta S, Manaktala U, Gupta S, Dubey $\mathrm{C}$, Khan M. Uterine tamponade using condom catheter balloon in the management of non-traumatic postpartum hemorrhage. J Obstet Gynaecol Res. 2012; 38(9):11627. doi: 10.1111/j.1447-0756.2011.01843.x. Epub 2012 Apr 30. PMID: 22540529. 


\section{TAPONAMIENTO UTERINO ARTESANAL CON CONDÓN E INNOVADOR SISTEMA DE DRENAJE \\ EN EL MANEJO DE HEMORRAGIA POSPARTO: A PROPÓSITO DE UN CASO}

6. Committee on Practice Bulletins-Obstetrics. Practice Bulletin No. 183: Postpartum Hemorrhage. Obstet Gynecol. 2017; 130(4):e168-e186. doi: 10.1097/ AOG.0000000000002351. PMID: 28937571.

7. Rodríguez J, Veroes J, González L, González M, Bello F. Uso del balón SOS Bakri en atonía uterina. Serie de casos en un hospital universitario de tercer nivel. Rev Obstet Ginecol Venez [Internet]. 2013 [consultado 22 de marzo de 2020]; 73(2):88-98. Disponible en: $\quad$ http://ve.scielo.org/scielo.php?script=sci arttext\&pid=S0048-77322013000200003

8. Fonseca-Chimá E. Balón de Bakri en hemorragia posparto en Medellín (Colombia): reporte de caso y revisión de la literatura. Rev Colomb Obstet Ginecol [Internet]. 2010 [consultado 22 de marzo de 2020]; 61(4):335-340. Disponible en: http://www.scielo.org. co/pdf/rcog/v61n4/v61n4a07.pdf
9. Pardo A, Gonzales M, Villarroel L. Dispositivo para la hemorragia puerperal (balón hemostático). Rev Cient Cienc Med [Internet]. 2013 [consultado 22 de marzo de 2020]; 16(1):32-34. Disponible en: http://www.scielo.org.bo/scielo.php? script $=$ sci arttext\&pid=S1817-74332013000100009

10. Mishra N, Gulabani K, Agrawal S, Shrivastava C. Efficacy and Feasibility of Chhattisgarh Balloon and Conventional Condom Balloon Tamponade: A 2-Year Prospective Study. J Obstet Gynaecol India. 2019; 69(Suppl 2):133-141. doi: 10.1007/s13224-0181185-6. Epub 2019 Jan 5. PMID: 31686746; PMCID: PMC6801252.

Recibido 19 de mayo de 2020 Aprobado 20 de septiembre de 2020 